\begin{tabular}{|c|c|c|}
\hline Beitr. Ent. & Berlin & ISSN 0005-805X \\
\hline $\mathbf{5 0}(2000) 1$ & S. $79-89$ & 11.04 .2000 \\
\hline
\end{tabular}

\title{
Die Arten der Anomala porovatula OHAUS-Gruppe von Sumatra
}

\section{(Coleoptera: Scarabaeidae: Rutelinae)}

\author{
Mit 11 Figuren
}

CARSTEN ZORN

\section{Zusammenfassung}

Das Typenmaterial von $A$. porovatula OHAUS, 1915 und $A$. personata OHAUS, 1932 wurde revidiert und Lectotypen für beide Arten designiert. Anomala exilis sp. n. and Anomala subita sp. n. werden neu von Sumatra beschrieben. Die abgehandelten Arten lassen sich in einer Artengruppe vereinigen, deren diagnostischen Merkmale zusammengefaßt werden. Die Aedoeagi aller Arten werden abgebildet.

\section{Summary}

The type material of $A$. porovatula OHAUS, 1915 and $A$. personata OHAUS, 1932 is revised and lectotypes are designated. Anomala exilis sp. n. and Anomala subita sp. n. are described from Sumatra. All the treated species are attributed to the same species group whose diagnostic characters are outlined. The male genitalia are figured.

\section{Key words}

Coleoptera, Scarabaeidae, Rutelinae, Anomala SAMOUELLE, taxonomy, new species, Oriental region, Sumatra

\section{Einleitung}

Im Jahr 1915 beschrieb OHAUS die Art Anomala porovatula von verschiedenen Fundorten auf Sumatra. Die Revision des Typenmaterials von Anomala porovatula OHAUS sowie die Bearbeitung von weiterem Anomala-Material von Sumatra ergab, daß bislang mehrere, teilweise außerordentlich ähnliche Arten unter diesem Namen vermengt wurden. Die Taxonomie sowie Verbreitung der mit Anomala porovatula OHAUS zunächst verwandten bzw. ihr ähnlichsten Arten soll daher Gegenstand dieser Arbeit sein. In diesem Zusammenhang wurde auch das Typenmaterial von $A$. personata OHAUS, 1932 revidiert, die ebenfalls diesem Artenkomplex angehört.

Für die hier abgehandelte Artengruppe können folgende diagnostische Merkmale zusammengefaßt werden:

- geringe Körpergröße (6,5-10,0 mm)

- einfacher Bau des Aedoeagus (einfache symmetrische Parameren, Ventralplatte ohne jegliche Anhänge) 
- Grundfarbe blaßgelb mit schwachem Erzglanz; gewöhnlich Tibien, Tarsen, Stirn und Scheitel bräunlich-erzgrün, die Mitte des Pronotum, Pygidium und Elytren mit verschiedengestaltigen bräunlich-erzgrünen Makeln, Elytren manchmal nahezu vollkommen verdunkelt

- Elytren mit regelmäßigen, gefurchten Punktreihen; die Punkte selbst ziemlich grob

- Rippen und Interstitien mit unregelmäßiger und weitläufiger Mikropunktur, sonst glatt und glänzend

- Pygidium mit groben, ocellierten Punkten, manchmal grob verrunzelt

- Metasternum lateral mit wenig dichter, ocellierter Punktur und ziemlich kurzer spärlicher Behaarung

- Protibien zweizähnig, größere Klaue der Pro- und Mesotarsen apical gespalten

Das im Text zitierte Material befindet sich in folgenden Sammlungen:

$\mathrm{MNHB}=$ Museum für Naturkunde der Humboldt-Universität zu Berlin

$\mathrm{BMNH}=$ The Natural History Museum, London

NHMB = Naturhistorisches Museum, Basel

CGF $=$ coll. Georg Frey im Naturhistorisches Museum, Basel

$\mathrm{CCZ}=$ coll. $\mathrm{C}$. ZORN (Dresden)

$\mathrm{CPH}=$ Phyllodrom, Institut und Museum für Regenwaldökologie, Leipzig

Das Material der neu beschriebenen Arten trägt folgendes rotes Etikett: „HOLOTYPUS (resp. PARATYPUS) Anomala exilis (resp. subita) sp. n. det. Zorn, 1999.““

\section{Anomala porovatula OHAUS, 1915}

(Fig. 1,2)

Anomala porovatula OHAUS, 1915, Stettin. ent. Ztg. 76: 142, f. 23. partim

Lectotypus: ơ "SUMATRA Palembang | Type | Anomala porovatula Ohs. | porovatula“ (MNHB). (hier designiert)

Paralectotypen: $10^{\pi}$ „Palembang Sumatra. Cotype | Anomala porovatula Ohs." (MNHB). 1 우 „Sumatra Drescher 4/1911 Kl. V. Annai | o | Cotype | Anomala porovatula Ohs." (MNHB) ( $=$ A. exilis sp. n.). $10^{*}$ - gleiche Angaben (ohne + -Zeichen) (MNHB) ( $=$ A. exilis sp. n.). $10^{*}$ „MEDAN Dolok Baros Estate Sumatra COLLECTION LE MOULT $\left|\sigma^{*}\right|$ Cotype $\mid$ Anomala porovatula Ohs." (MNHB) (=A. exilis sp. n. ). 1 ㅇ - gleiche Angaben (ohne $0^{*}$-Zeichen) $(\mathrm{MNHB})(=$ A. exilis sp. n. ). 1 ㅇ ,Sumatra $|+|$ Cotype | Anomala porovatula Ohs." (MNHB) (=A. exilis sp. n.) (hier designiert).

Bemerkungen: OHAUS vermengte unter der Typenserie mehr als eine Art. Die letzten 5 zitierten Exemplare der Typenserie gehören zu A. exilis sp. n., die weiter unten beschrieben wird. Die im folgenden zitierten und von OHAUS als „Cotype" gekennzeichneten Exemplare besitzen keinen Typenstatus, da sie in der Originalbeschreibung nicht erwähnt werden: $10^{*}$ „Sumatra Padang $\mid$ Cotype $\mid$ Anomala porovatula Ohs." (MNHB) (=Anomala subita sp. n.). 1 i "Sumatra Padang $|q|$ Cotype | Anomala porovatula Ohs." (MNHB) (=Anomala subita sp. n.). 1 ㅇ „Sumatra Barisan Geb |400' D. Behrens. I / Cotype / Anomala porovatula Ohs." (MNHB). (=A. subita sp. n.). $10^{\prime}$, KANNEGIETER Ins.Batoe.9'96 Tanah Masa | Cotype | Anomala porovatula Ohs." (MNHB) (=A. subita sp. n.). 10" „Sumatra Deyr.[?] $\left|\sigma^{\pi}\right|$ Cotype | Anomala porovatula Ohs." (MNHB) (=A. personata OHAUS, 1932).

Weiteres Material: $10^{\pi}$ „Lebong Tandai, W. Sumatra. 1921. C. J. Brooks coll. | C.J.Brooks. B.M.1936-681. | Ohaus determ. Anomala porovatula Ohs. $\sigma^{\text {"6 }}$ (BMNH). 10" „Lebong Tandai, W. Sumatra. 8 - 11.VII.1922. C. J. Brooks coll. No. 7785|C.J.Brooks. B.M.1936-681. |Ohaus determ. Anomala porovatula Ohs. $\sigma^{\text {" }}$ (BMNH). $30^{\prime \prime}$ „Palembang Sumatra | Nevinson Coll. 
1918-14." (BMNH). 70, 2 ㅇ „Sumatra Palembang“(MNHB). 1 \% „Bedagei. int. Sumatra's O.K. $600^{\prime}$ 2. e Sem. 89 I. Z. Kannegieter" (MNHB). 40", 7 ㅇ „Indonesia Sumatra Barat, Padang Pariaman, leg. Widagdo" (CCZ). 120", $5 \circ$,Indonesia Sumatra Barat, Harau Valley, ca. 400m, leg. Bujang" (CCZ).

\section{Beschreibung}

Körperumriß: Länge: 7,0-8,1 mm ( $\left.\sigma^{7}\right), 7,7-9,1 \mathrm{~mm}($ ( ) $)$ Breite: 4,2-4,7 mm ( $\left.\sigma^{\pi}\right), 4,3-5,2 \mathrm{~mm}$ ( $)$; oval; nach hinten verbreitert; mäßig gewölbt.

Färbung: Grundfarbe blaßgelb mit schwachem Erzglanz; Stirn, Scheitel, Pronotum außer den gelben Seiten, das Scutellum in unterschiedlicher Ausdehnung, der überwiegende Teil der Elytren, Basis des Pygidium sowie Tibien und Tarsen erzgrün, bräunlich durchscheinend. Elytren mit strichförmigen gelben Makeln im Bereich der zweiten primären Rippe sowie hinter und neben dem Humeralkallus sowie entlang des Apicalrandes; Abdominalsternite braun getrübt. Weibchen weichen in der Färbung etwas ab (siehe unten).

Caput: Clypeus quer trapezoid mit verrundeten Vorderecken und aufgebogenem Rand; Punktur äußerst dicht und teilweise verrunzelt; Clypeofrontalnaht gerade; Stirn wie der Clypeus, Scheitel nach posterior zunehmend feiner und einzeln punktiert; Fühlerkeule kürzer als die übrigen Glieder zusammen, aber nur wenig länger als der Funiculus.

Pronotum: ziemlich quer, ca. 1,7x so breit wie lang; an der Basis am breitesten; nach vorn in der posterioren Hälfte nur schwach, im anterioren Teil stark und gerade konvergierend; Vorderwinkel scharf rechtwinklig, deutlich vorgezogen; Hinterwinkel dagegen stumpf verrundet; basale Randfurche vollständig; Punktur in der Mitte fein, lateral ziemlich grob, mit teilweise sehr unregelmäßigen Abständen; Seitengrübchen undeutlich.

Scutellum: mit feiner, wenig dichter Punktur; Hinterrand punktfrei.

Elytren: mit gefurchten, grob punktierten Punktreihen; sekundäre Punktreihen im subsuturalen, zweiten und dritten Interstitium; erstere nach vorn unregelmäßig verdoppelt; alle Rippen und Interstitien gewölbt, mit Mikropunktur und stark glänzend; hinter dem Humeralkallus einige Querfältchen.

Pygidium: grob und dicht punktiert; Abstände unregelmäßig; Punkte manchmal quer ineinanderfließend; entlang des Distalrandes mit einzelnen abstehenden Saeten.

Metasternum: mit grober, ocellierter Punktur, die auch lateral nur selten ineinanderfließt; mit greiser, kurzer Behaarung.

Abdominalsternite: grob und dicht punktiert; mit der gewöhnlichen queren Borstenreihe; Distalrand des letzten Sternit mit sehr schwacher Ausrandung in der Mitte.

Protibien: zweizähnig; Lateralzahn deutlich, zumeist rechtwinklig; Terminalzahn mäßig lang, nach außen gekrümmt.

Metatibien: ca. 2,8x so lang wie breit; etwas wadenartig verdickt und apical eingeschnürt.

Klauen: größere Klaue der Pro- und Mesotarsen an der Spitze gespalten; erstere unterseits etwas erweitert, aber nicht gewinkelt oder gezahnt.

Weibchen: durchschnittlich etwas größer (siehe oben); helle Färbung der Elytren oft stärker ausgedehnt, zusammenhängende dunkle Zeichnung manchmal nur noch hinter dem Scutellum, entlang der Suturalnaht sowie hinter dem Humeralkallus; Seiten des Pronotum stärker gerundet; Distalrand des letzten Abdominalsternit einfach; Terminalzahn der Protibien etwas länger und stumpfer; größere Klaue der Protarsen schlank.

Bionomie und Verbreitung: Die Verbreitung dieser Art scheint auf den mittleren bis südlichen Teil Sumatras beschränkt zu sein. Die nördlichsten Funde an der Westküste liegen in der Gegend um Padang Panjang (etwa $60 \mathrm{~km}$ nördlich von Padang). Die Verbreitung der Art im Osten der Insel ist ungeklärt. 
Angaben zur Lebensweise liegen nicht vor. Die Fundortangaben reichen höhenzonal vom Tiefland bis zum submontanen Bereich.

Diagnose: Die Originalbeschreibung bezog sich im wesentlichen auf die tatsächliche $A$. porovatula OHAUS. Nur bei der Darstellung vermeintlich variabler Merkmale wurden offensichtlich Exemplare von $A$. exilis sp. n. von OHAUs (1.c.) zugrunde gelegt. Das Pronotum besitzt wie oben beschrieben außer den gelben Seiten keine weiteren hellen Makeln. Die Erwähnung gelber Flecken vor dem Scutellum sowie einer gelben Mittellinie bezieht sich auf fälschlicherweise hier eingeordnete Exemplare von A. exilis sp. n. Aufgrund dieses Merkmals ist A. porovatula OHAUS fast immer gut von der folgenden Art zu unterscheiden. Auch die Punktur des Pronotum ist bei $A$. porovatula OHAUS gröber und unregelmäßiger.

\section{Anomala exilis sp. $\mathbf{n}$.}

(Fig. 3, 4, 5, 6)

Anomala porovatula OHAUS, 1915, Stettin. ent. Ztg. 76: 142, f. 23. part. syn.

Holotypus: o" „SUMATRA: 12 km. N of Bukittinggi, Bukit Palupuh, 1000m., 19.-24.x.1980. | A. Harman Coll. B. M. 1982-49." (BMNH).

Paratypen: $10^{*}$ „MEDAN Dolok Baros Estate Sumatra COLLECTION LE MOULT | $\sigma^{*}$ | Cotype | Anomala porovatula Ohs." (MNHB). 19 gleiche Angaben (ohne $\sigma^{x}$-Zeichen) (MNHB). 19 ,Sumatra $|+|$ Cotype | Anomala porovatula Ohs." (MNHB). $10^{7}$ "Indonesia Sumatra Aceh Kuala Dou bei Calang leg. Zorn" (CCZ). 2 ㅇ - gleiche Angaben (CCZ). $10^{7}$ „Aek Tarum 4.VI.1978 | Sumatra Dr. E.Diehl“ (NHMB). 10" „Solok Sumatra EX COLL. F. SCHNEIDER“ (MNHB). 19 ,J. B. CORPORAAL Sumatra's O. K. Sidoemanik. 14.2.|22|.| q " (MNHB). $10^{\star}$ „Sumatra. J. B. Corporaal. B. M. 1924-480.| J. B. CorporaAl Sumatra's O. K. Siantar. 8-8-1921 Sidoemanik 400 M/det.OHAUS 1922 Anomala porovatula Ohs. "(BMNH). $10^{7}$ „Sumatra Merang | Doherty | Fry Coll. 1905-100.“ (BMNH). 1 \% ,67166 | Doherty | Sumatra Merang | Fry Coll. 1905-100." (BMNH). 1 \% „Sumatra Drescher 4/1911 Kl. V. Annai | | Cotype | Anomala porovatula Ohs." (MNHB). 1 우 - gleiche Angaben (ohne 9 -Zeichen) (MNHB). 10" ,Fort de Kock (Sumatra) 920M. 1925 leg. E. Jacobson. | $0^{7} \mid$ Anomala porovatula Ohs." (MNHB). 1 우 „Fort de Kock (Sumatra) 920M. 1925 leg. E. Jacobson/Ohaus determ. Anomala porovatula Ohs." (MNHB). 10" „Fort de Kock, W. Sumatra. E.Jacobson. B.M.19262. Anomala porovatula Ohs. $\sigma^{\pi}$. det. Ohaus" (BMNH).

\section{Beschreibung}

Körperumriß: Länge: 6,5-8,6 mm, Breite: 3,7-4,6 mm; Körper kurz oval, mäßig gewölbt. Färbung: Grundfarbe blaßgelb mit schwachem Erzschimmer; Stirn, Scheitel und Pronotum bis auf die breit gelb gesäumten Seiten, eine schmale gelbe Mittellinie sowie zwei kleine schräg gestellte gelbe Basalmakeln braun mit grünem Erzglanz; Basalmakeln und helle Mittellinie fehlen nur im Ausnahmefall; Elytren, bis auf längsstreifige gelbe Makeln im Bereich der zweiten primären Rippe sowie innen neben und hinter dem Humeralcallus, völlig braun mit erzgrünem Glanz; Vorderrand des Pygidium braun mit Erzlanz; Tibien und Tarsen bräunlich erzgrün.

Caput: Clypeus quer trapezoid mit verrundeten Vorderecken, Vorder- und Seitenrand stark aufgebogen; Punktur vorn kräftig und einzeln, nach hinten quer ineinanderfließend; Clypeofrontalnaht gerade; Stirn mit quer verrunzelter Punktur; Scheitel einzeln, nach hinten zunehmend feiner punktiert; Fühlerkeule kürzer als die übrigen Glieder zusammen, etwas länger als der Funiculus. 

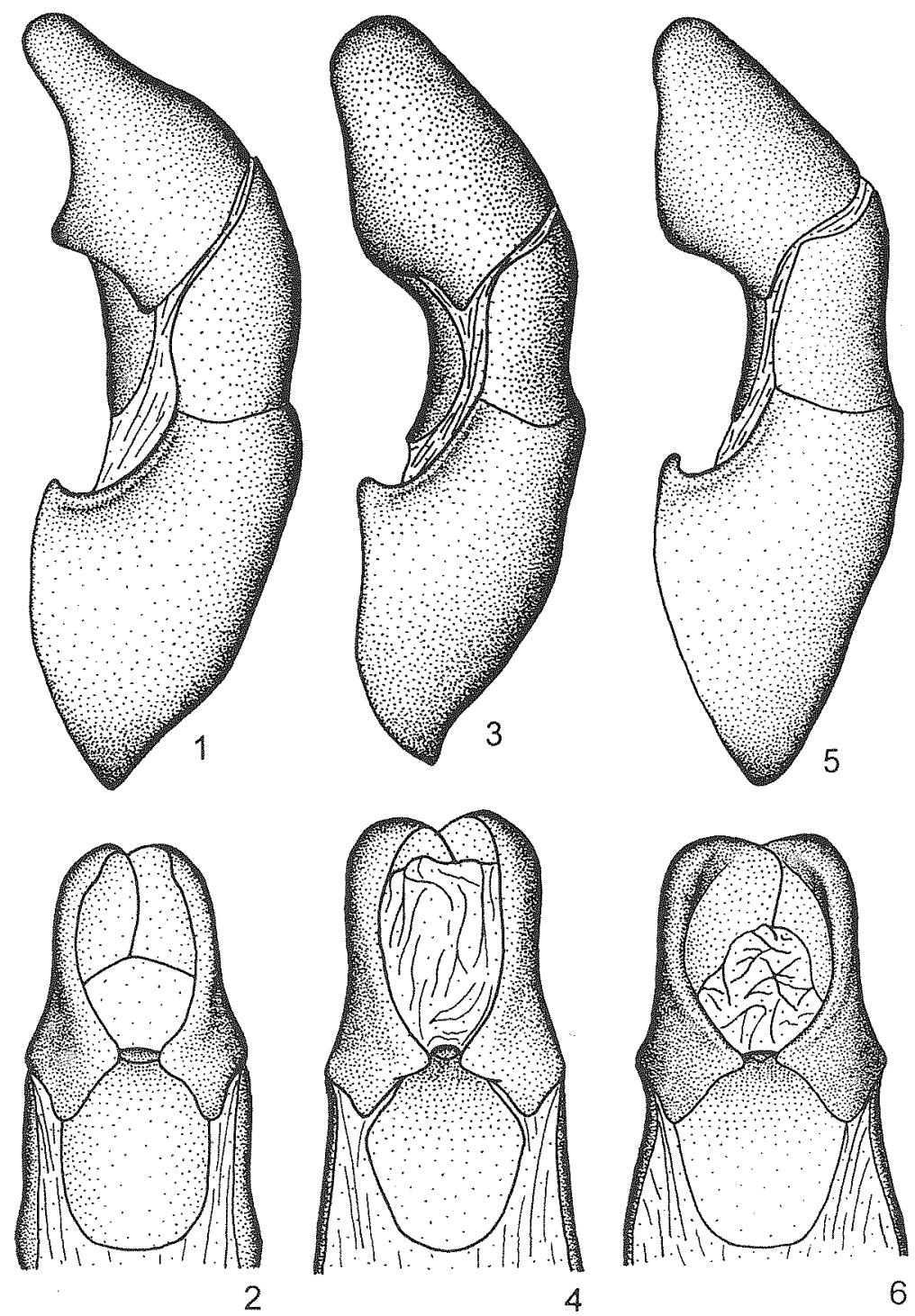

Fig. 1-2. Anomala porovatula OHAUs (Lectotypus: Sumatra, Palembang). Fig. 1, Aedoeagus lateral; Fig. 2, Aedoeagus ventral; Fig. 3-4. Anomala exilis sp. n. (Paratypus: Sumatra, Medan). Fig. 3, Aedoeagus lateral; Fig. 4, Aedoeagus ventral; Fig. 5-6. Anomala exilis sp. n. (Holotypus: Sumatra, Bukittinggi). Fig. 5, Aedoeagus lateral; Fig. 6, Aedoeagus ventral. Maßstab $=1 \mathrm{~mm}$.

Pronotum: ziemlich stark quer, ca. 1,7x mal so breit wie lang; die größte Breite an der Basis; nach vorn in gleichmäßiger Rundung verengt; Vorderwinkel spitz und vorgezogen; Hinterwinkel stumpf, abgerundet; basale Randfurche vollständig; Punktur mäßig fein bis mäßig grob, diese discal zerstreut (Abstände zwischen den Punkten 2-5x so groß wie ihr Durchmesser) und lateral etwas dichter; Seitengrübchen undeutlich.

Scutellum: etwas feiner als das Pronotum punktiert. 
Elytren: mit grob punktierten, kräftig gefurchten Punktreihen; die Rippen und Interstitien etwas gewölbt; sekundäre Punkreihen befinden sich im subsuturalen, zweiten und dritten Interstitium; erstere ist nach vorn unregelmäßig verdoppelt; Rippen und Interstitien mit zusätzlicher zerstreuter Mikropunktur.

Pygidium: mit großer, flacher, teilweise ineinanderfließender, ocellierter Punktur; an der Spitze sowie im Bereich der Vorderwinkel mit einzelnen abstehenden Saeten.

Metasternum: im lateralen Bereich mit einzelner, grober, ocellierter Punktur und spärlicher, kurzer heller Behaarung.

Abdominalsternite: fein und mäßig dicht punktiert; mit deutlicher Borstenreihe; Distalrand des letzten Sternit in der Mitte etwas ausgerandet.

Protibien: zweizähnig; Lateralzahn kräftig und spitz; Terminalzahn lang und nach außen gebogen, ziemlich spitz.

Metatibien: etwas verdickt; ca. $2,8 \mathrm{x}$ so lang wie breit; apical eingeschnürt.

Klauen: größere Klaue der Pro- und Mesotarsen an der Spitze gespalten, erstere unterseits etwas erweitert, aber nicht gewinkelt oder gezahnt.

Weibchen: sekundäre Geschlechtsmerkmale nur schwach ausgeprägt; Distalrand des letzten Sternit einfach; Terminalzahn der Protibien etwas stumpfer und weniger stark nach außen gebogen; größere Klaue der Protarsen schlank.

Bionomie und Verbreitung: Anomala exilis sp. n. scheint nur den nördlichen und mittleren Teil Sumatras zu besiedeln. Alle Fundorte befinden sich in den Provinzen Aceh, SumatraUtara, Sumatra-Barat und Jambi. Die Areale von $A$. exilis sp. n. und $A$. porovatula OHAUS überlappen sich also in Mittelsumatra. Die Fundortangabe „K1. v. Annan“ konnte allerdings nicht lokalisiert werden.

Im MNHB befindet sich ein identisches Exemplar mit der Fundortangabe West-Java (욱). Hierbei handelt es sich, wenn nicht um eine Fundortsverwechslung, möglicherweise um eine distinkte Art. Das Exemplar wurde aufgrund dieser Unsicherheit nicht in die Typenserie dieser Art miteinbezogen.

Alle Funde stammen höhenzonal aus dem Bereich des Tieflandsregenwaldes bzw. submontanen Regenwaldes. Anomala exilis sp. n. wurde mehrfach am Licht (250 W Mischlicht) gefangen und ist daher offensichtlich nachtaktiv.

Diagnose: Anomala exilis sp. n. ist $A$. porovatula OHAUS außerordentlich ähnlich. Neben konstanten Unterschieden im Bau des Aedoeagus läßt sie sich aber anhand des Auftretens der zwei gelblichen Makel am Basalrand des Pronotum bzw. der hellen Mittellinie in den allermeisten Fällen sicher von dieser trennen. Diese Makeln fehlen bei A. porovatula OHAUS grundsätzlich, bei Anomala exilis sp. n. sind sie dagegen fast immer vorhanden. Die Punktur auf dem Pronotum ist feiner. Wie die Figuren 3-6 verdeutlichen, ist der Paramerenbau nicht einheitlich. Exemplare mit längeren Parameren von nordwestlich gelegenen Fundorten und Tiere mit kürzeren Parameren vor allem aus Sumatra-Barat scheinen auf Differenzen hinzuweisen, die möglicherweise von taxonomischer Bedeutung sind, doch besitzen nicht alle Exemplare von Sumatra-Barat und Jambi kürzere Parameren und Übergänge sind vorhanden. Die Untersuchung zusätzlichen Materials ist notwendig, um diesen Sachverhalt eindeutig bewerten zu können.

\section{Anomala personata OHAUS, 1932}

(Fig. 10, 11)

Anomala personata OHAUs, 1932, Ann. Mag. Nat. Hist. 10 (9): 406, f. 1.

Lectotypus: $\sigma^{*}$,Type / Sumatra: Mt. Dempo, 4000 ft. 1920-23 C.J.Brooks. | Ohaus determ. Anomala personata Type o* Ohs." (BMNH). (hier designiert) 
Paralectotypen: $1 \sigma^{7}$ „Cotype | Sumatra: Mt. Dempo, 4000 ft. 1920-23 C.J.Brooks. | Ohaus determ. Anomala personata Cotype o Ohs." (BMNH). 1 \% „Cotype / Sumatra: Mt. Dempo, $4000 \mathrm{ft}$. 1920-23 C.J.Brooks. Ohaus determ. Anomala personata Cotype 9 Ohs." (BMNH). 1 ㅇ „Sumatra: Mt. Dempo, $4000 \mathrm{ft} .1920-23$ C.J.Brooks. | Anomala personata Ohs." (MNHB). 1 ㅇ „Sumatra: Mt. Dempo, 4000 ft. 1920-23 C.J.Brooks. I \& / Cotype | Anomala personata Ohs." (MNHB). (hier designiert)

Weiteres Material: $10^{*}$ "Sumatra Deyr.[?] $\mid \sigma^{*}$ Cotype / Anomala porovatula Ohs." (MNHB). 1 오 „W. Sumatra. Aug. 1923 C. J. Brooks coll. No. 15448 Dempo 4000'|15448'|C.J.Brooks. B.M. 1936-681" (BMNH). 10", 2ㅇ „Sumatra Deyr. v. [?]" (MNHB). 10" „Java Buitenzorg“ (MNHB). [?]1 ${ }^{\circ}$, ,SUMATRA(W.), G. Singgalang S of BUKITTINGGI 14.-16.II.1991, $1300 \mathrm{~m}$ " (CCZ).

Bemerkungen: Eine Lectotypendesignierung wurde vorgenommen, da nicht zweifelsfrei auszuschließen ist, daß weitere, eventuell nicht zu dieser Art gehörende Exemplare der Typenserie existieren.

\section{Beschreibung}

Körperumriß: Länge: 8,5-10,0 mm, Breite: 4,6-5,3 mm; lang oval; nach hinten verbreitert; ziemlich flach gewölbt.

Färbung: Grundfarbe blaßgelb mit schwachem Erzschimmer; Stirn, Scheitel, Pronotum bis auf die breit gelb gerandeten Seiten, eine halb ellipsoide Makel hinter dem Scutellum sowie ein kleiner Fleck unter dem Humeralkallus auf den Elytren sowie Tibien und Tarsen braun mit erzgrünem Glanz; Scutellum nur in Ausnahmefällen verdunkelt; meist auch Abdominalsternite, Basis des Pygidium und Seiten des Metasternum braun-erzgrün getruibt.

Caput: Clypeus quer trapezoid mit abgerundeten Vorderecken; Rand aufgebogen; Clypeofrontalnaht gerade; Punktur von Clypeus und Stirn dicht und teilweise verrunzelt; Scheitel nach posterior zunehmend feiner und einzeln punktiert; Fühlerkeule kürzer als die übrigen Glieder zusammen, aber deutlich länger als der Funiculus.

Pronotum: ziemlich stark quer, ca. 1,8x so breit wie lang; größte Breite an der Basis; Seiten nach anterior zunächst parallel, in der Mitte gebogen und vor den Vorderwinkeln gerade und stark verengt; Vorderwinkel scharf rechtwinklig; Hinterwinkel stumpf und nur wenig abgerundet; basale Randfurche vollständig; Punktur in der Mitte etwas feiner, lateral gröber und unregelmäßig ineinanderfließend; Mittellinie des Pronotum mehr oder weniger deutlich gefurcht. Scutellum: mäßig fein und dicht punktiert.

Elytren: mit regelmäßigen, gefurchten Punktstreifen; sekundäre Punktreihen im zweiten und dritten Interstitium; die gröbere Punktur des subsuturalen Interstitium formt sich erst apical zu einer regelmäßigen Punktreihe; auf allen Rippen und Interstitien mit weitläufiger Mikropunktur. Pygidium: mit groben Punkten, die nur selten ineinanderfließen und dann zumeist quere Runzeln zeigen; mit einzelnen Saeten im Bereich des Distalrandes.

Metasternum: mit grober, ocellierter Punktur, die auch lateral nicht ineinanderfließt; mit greiser, wenig langer Behaarung.

Abdominalsternite: mäßig dicht, aber einzeln punktiert; mit querer Borstenreihe; Distalrand des letzten Sternit in der Mitte ausgerandet.

Protibien: zweizähnig; Terminalzahn sehr kräftig und stark nach außen gebogen; Lateralzahn relativ klein, etwas spitzwinklig.

Metatibien: ausgesprochen schlank, ca. 3,6x so lang wie breit und apical deutlich eingeschnürt. Klauen: größere Klaue der Meso- und Protarsen apical gespalten; die der Protarsen am Innenrand erweitert, aber nicht gewinkelt oder gezahnt.

Weibchen: Statur etwas robuster, Seiten des Pronotum stärker gerundet; Distalrand des letzten Abdominalsternit nicht ausgerandet; Metatibien robuster, apical stärker verbreitert; Terminalzahn der Protibien sehr lang, leicht spatelförmig verbreitert und apical nach außen gebogen; größere Klaue der Protarsen schlank. 
Bionomie und Verbreitung: Die Art ist bislang nur vom Gunung Dempo (,Berg Dempo von Südwestsumatra (Provinz Sumatra Selatan) sowie von West-Java (Buitenzorg = Bogor) bekannt. Das Exemplar vom Gunung Singgalang weicht morphologisch etwas ab, es ist etwas größer und höher gewölbt, die dunkle Zeichnung ist rein erzgrün, und auch die Punktstreifen der Elytren sind dunkel unterlegt. Möglicherweise handelt es sich hierbei um eine eigene Art bzw. um eine Subspecies von $A$. personata OHAUS.

Die Höhenangaben auf den Etiketten weisen auf eine submontane bis montane Lebensweise von A. personata OHAUS hin.

Diagnose: Anomala personata OHAUS ist vor allem A. tenimbrica OHAUS, 1936 außerordentlich ähnlich, die von den südöstlichen Molukken (Insel Tanimbar) beschrieben wurde. Allein das Pronotum ist bei dieser nur im Holotypus bekannten Art etwas stärker gerunzelt und der Aedoeagus ist kleiner, sonst jedoch nahezu identisch. Interessanterweise liegen die Areale beider Arten voneinander weit entfernt, und verwandte Arten scheinen auf den sich zwischen Java und Tanimbar erstreckenden kleinen Sunda-Inseln völlig zu fehlen.

Innerhalb der sumatranischen Arten der $A$. porovatula-Gruppe ist $A$. personata OHAUS aufgrund ihrer länglichen, flach gewölbten Körpergestalt, der großen dunklen Pronotummakel bei sonst überwiegend hellen Elytren leicht identifizierbar. Der Aedoeagus ist, auch in Relation zur Körperlänge, deutlich größer als bei den übrigen Arten. Eine nähere Verwandtschaft zu $A$. decorata KIRSCH, 1875, wie bei OHAUS (1932) erwähnt, besteht nicht.

\section{Anomala subita sp. n.}

(Fig. 7,8,9)

Holotypus: $\sigma^{\pi}$ „Sumatra Padang / Cotype / Anomala porovatula Ohs.“ (MNHB).

Paratypen: 19 "Sumatra Padang | o | Cotype | Anomala porovatula Ohs." (MNHB). 1 \% "Sumatra Barisan Geb | 400' D. Behrens. I | Cotype | Anomala porovatula Ohs." (MNHB). $10^{\prime \prime}$ „Indonesia Sumatra - Barat Umg. Padang Panjang 700-1000 m 21.I. - 25.I.95 leg. C.Zorn" (CCZ). 1 ㅇ „Indonesien, West-Sumatra GUGUK 1991 leg. Widagdo" (CPH). $1 \%$,INDONESIA Sumatra Barat, Lembah Anai Resort Kandang Ampek ca. $15 \mathrm{~km}$ SE Padang Panjang, ca. 500m III. 1997 leg. C.u.P.Zorn" (CCZ). 1 of „Buitenzorg Java / cum Typo comparatum | Anomala personata Ohs.“ (CGF). 19 „KANNEGIETER Ins.Batoe.9'96 Tanah Masa | Cotype | Anomala porovatula Ohs." (MNHB).

\section{Beschreibung}

Körperumriß: Länge: 9,0-10,0 mm, Breite: 5,3-5,8 mm; länglich oval; nach hinten schwach erweitert; mäßig gewölbt.

Färbung: hell blaßgelb mit sehr schwachem Erzglanz; Stirn, Scheitel, Tibien und Tarsen sowie Abdominalsternite braun mit grünlichem Erzglanz; Pronotum mit scharf umrissener, innen brauner, lateral geschwärzt-erzgrüner Makel auf dem Diskus; diese am Vorderrand etwa so breit wie die Stirn zwischen den Augen, nach posterior zunächst verbreitert und hinter scharf ausgeprägten Seitenecken spitz zusammenlaufend; hintere Spitze der Makel etwas eingeschnitten, erreicht nicht den Basalrand des Pronotum; Elytren mit strichförmiger dunkler Makel unterhalb des Humeralkallus sowie bogenförmiger Discoidalmakel direkt hinter dem Scutellum; letztere innen braun, am Außen- und Hinterrand geschwärzt und mit Erzglanz; nur beim Exemplar von Batu Elytren insgesamt braun verdunkelt; dabei durch dunkler braune Interstitien etwas streifenförmig gezeichnet.

Caput: Clypeus quer trapezoid mit abgerundeten Vorderecken; Vorderrand kräftig aufgebogen; mit sehr dichter, zum Teil quer zusammenfließender Punktur; Clypeofrontalnaht in der Mitte leicht nach posterior geschwungen; Stirn wie der Clypeus, Scheitel fein und zerstreut punktiert; Fühlerkeule etwas länger als der Funiculus. 

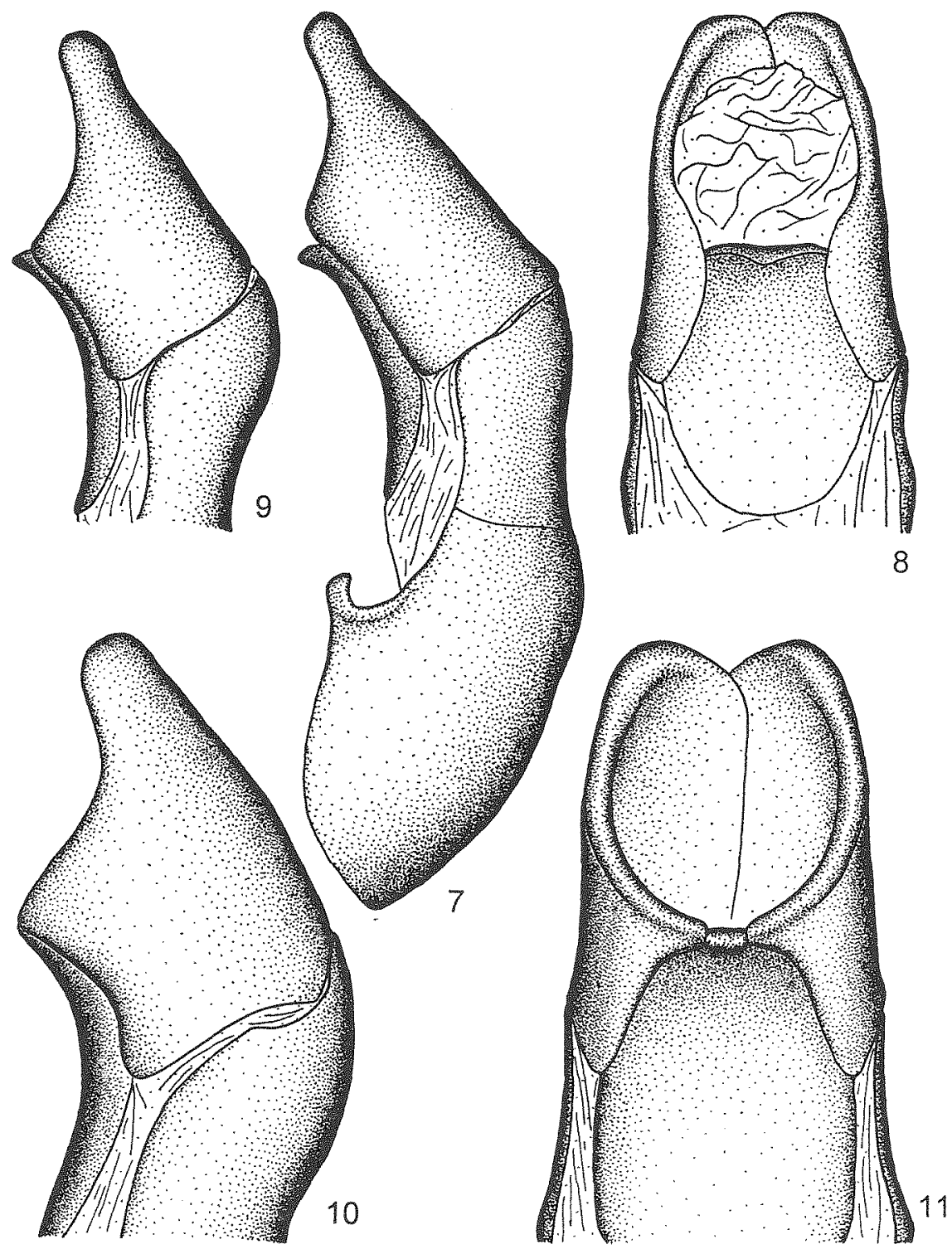

Fig. 7-8. Anomala subita sp. n. (Holotypus: Sumatra, Padang). Fig. 7, Aedoeagus lateral; Fig. 8, Aedoeagus ventral; Fig. 9. Anomala subita sp. n. (Paratypus: Insel Batu), Aedoeagus lateral; Fig. 10-11. Anomala personata OHAus (Paralectotypus: Sumatra, Mt. Dempo). Fig. 10, Aedoeagus lateral; Fig. 11 , Aedoeagus ventral. Maßstab $=1 \mathrm{~mm}$.

Pronotum: quer, ca. 1,6x so breit wie in der Mitte lang; an der Basis am breitesten; Seiten von den stumpfen, schwach abgerundeten Hinterwinkeln nach anterior zunächst fast parallel, in der vorderen Hälfte dann stark und fast gerade konvergierend; Vorderwinkel spitz- bis recht- 
winklig, deutlich vorgezogen; basale Randfurche vollständig; Punktur in der Mitte fein, nach lateral zerstreuter und deutlich gröber, nur unmittelbar am Lateralrand wieder etwas dichter; Abstände zwischen den Punkten überall ziemlich unregelmäßig; Seitengrübchen zumeist undeutlich.

Scutellum: extrem fein punktiert.

Elytren: mit grob punktierten, deutlich gefurchten regelmäßgen Punktreihen; Rippen und Interstitien daher etwas gewölbt; sekundäre, weniger tief gefurchte, hinten verkürzte Punktreihen im zweiten und dritten Interstitium; subsuturales Interstitium mit unregelmäßiger Punktur, die sich erst am Apex zu einer Punktreihe formt; alle Rippen und Interstitien mit zerstreuter unregelmäßiger Mikropunktur; im Bereich des zweiten Interstitium mit deutlichen Querfältchen.

Pygidium: mit grober ocellierter Punktur; diese teilweise zusammenfließend, so daß eine grobrunzlige Struktur entsteht; am Distalrand mit einzelnen abstehenden Saeten.

Metasternum: mit groben ocellierten Punkten, die auch lateral zumeist deutlich getrennt sind; helle Behaarung ziemlich spärlich und kurz.

Abdominalsternite: in der Mitte etwas feiner und zerstreuter, nach lateral dicht und grob punktiert; quere Bostenreihe der vorderen Sternite undeutlich; Distalrand des letzten Sternit in der Mitte tief ausgerandet.

Protibien: zweizähnig, Lateralzahn spitz und kräftig, Terminalzahn schräge nach außen gebogen.

Metatibien: ca. 3,1x so lang wie breit; schwach wadenartig verbreitert und apical etwas eingeschnürt.

Klauen: größere Klaue der Pro- und Mesotarsen apical gespalten; die der Protarsen unterseitig schwach verbreitert.

Weibchen: Pronotum etwas stärker gewölbt; Distalrand des letzten Abdominalsternit einfach; Terminalzahn der Protibien etwas länger und apical spatelartig verbreitert; größere Klaue der Protarsen schmal.

Bionomie und Verbreitung: Die Art ist bislang aus der Provinz West-Sumatra, von der Insel Batu und West-Java bekannt, wo sie vor allem den submontanen Bereich zu besiedeln scheint.

Diagnose: Anomala subita sp. n. ist aufgrund ihrer überwiegend hellgelben Färbung und der typischen dunklen Zeichnung $A$. personata OHAUS und $A$. tenimbrica OHAUS ähnlich. Letztere sind aber durchschnittlich etwas größer und verhältnismäßig schlanker, die Pronotummakel ist hinten nicht verschmälert und reicht bis zum Basalrand. Der Aedoeagus ist bei beiden Arten sehr ähnlich gestaltet, aber deutlich größer. Die Variationsbreite des Aedoegusbau bei $A$. subita sp. n. verdeutlichen die Figuren 7-9.

Das Exemplar von der Insel Batu weicht hinsichtlich der Körperfärbung wie oben beschrieben von den übrigen Exemplaren ab. Die Form des Aedoeagus dagegen liegt innerhalb der Variationsbreite der Tiere von Sumatra.

\section{Bestimmungstabelle für die Anomala porovatula - Artengruppe von Sumatra}

1 die dunkle Zeichnung des Pronotum nach hinten verschmälert, nicht den Basalrand erreichend $\ldots \ldots \ldots \ldots \ldots \ldots \ldots \ldots \ldots \ldots \ldots \ldots \ldots \ldots \ldots \ldots \ldots \ldots$ subita sp. $\mathrm{n}$. die dunkle Zeichnung des Pronotum nach hinten verbreitert, den Basalrand des Pronotum erreichend $\ldots \ldots \ldots \ldots \ldots \ldots \ldots \ldots \ldots \ldots \ldots \ldots \ldots$

2. größere Art von 8,5-10 mm Körperlänge; flach gewölbt; Elytren überwiegend blaßgelb mit halbellipsoider Makel hinter dem Scutellum ........ A. personata OHAUS 
- $\quad$ kleinere Arten von 6,5-8,6 mm Körperlänge; mäßig hoch gewölbt; Elytren immer bräunlich-erzgrün mit streifenförmigen gelblichen Makeln $\ldots \ldots \ldots \ldots \ldots$

3. erzgrüne Makel des Pronotum weder vor der Basis noch entlang der Mitte von gelblichen Aufhellungen durchsetzt, Punktur des Pronotum etwas gröber (Prov. Sumatra Barat, Jambi, Sumatra Selatan) ............ A. porovatula OHAUS Pronotum mit aufgehellter Mittellinie sowie mit zwei kleinen, schräggestellten gelben Makeln vor der Basis; diese selten undeutlich oder fehlend; manchmal fast die gesamte Basis hell, Pronotumpunktur auf dem Diskus \pm fein (Prov. Aceh, Sumatra Utara, Sumatra Barat, Jambi) $\ldots \ldots \ldots \ldots \ldots \ldots \ldots \ldots \ldots \ldots \ldots \ldots \ldots \ldots \ldots \ldots \ldots$ exilis $\mathrm{sp} . \mathrm{n}$.

\title{
Danksagung
}

Mein herzlichster Dank gilt den Herren Dr. M. UHLIG, B. JAEGER und J. SCHULzE (MNHB), Herrn Dr. M. BrancucCI und Frau Dr. E. Sprecher (NHMB) sowie Mr. M. KerLeY (BMNH), die mich freundlich unterstützten, indem sie mir Zugang zu Käfermaterial und insbesondere den in den entsprechenden Museen aufbewahrten Typen ermöglichten.

\section{Literatur}

KIRSCH, TH. 1875: Neue Käfer aus Malacca. - Mitt. Zool. Mus. Dresden 1: 26-58.

OHAUS, F. 1915: XVII. Beitrag zur Kenntnis der Ruteliden (Col. lamell.). - Stettin. Ent. Ztg. 76: 88-143. OHAUS, F. 1932: Two new Species of Anomala from British N. Borneo and Sumatra in the British Museum. - Ann. \& Mag. Nat. Hist. 10 (9): 405-406.

OHAUS, F. 1936: XXIX. Beitrag zur Kenntnis der Rutelinae (Col. Scarab.). - Stettin. Ent. Ztg. 97: 141-153.

\author{
Anschrift des Verfassers: \\ CARSTEN ZORN \\ Kamenzer Straße 11 \\ D - 01099 Dresden \\ Deutschland \\ e-mail: czorn@hotmail.com
}

\section{Besprechungen}

LOHMANN, M.: Vögel am Futterhaus: Vögel in Gärten und Parks erkennen und richtig füttern. München; Wien; Zürich: BLV Verlagsgesellschaft, 1999. - 95 S.: zahlr. Abb. - (BLV Naturführer). ISBN 3-405-15118-X. - 12.90 DM

Es muß nicht immer (Meisen-)Knödel sein. Wie man dem gefiederten Volk im Winter den eigenen Garten schmackhaft macht, beschreibt MICHAEL LOHMANN im BLV Naturführer Vögel am Futterhaus und verrät dabei den Naturfreund ganz erstaunliche Tricks und praktische Tipps für eine abwechslungsreiche Speisekarte. Da fehlen weder Körnchen vom Feinsten und beerenstarke Kost für Meisen, Finken, Kernbeißer und sogar den Seidenschwanz, noch tierische Leckerbissen für seltene Wintergäste wie Zaunkönige und Rotkehlchen. Und mit dem Bestimmungsteil lassen sich die geladenen und gern gesehenen Gäste auch gleich bestens erkennen. 85 brillante Farbfotos zeigen die Vögel im Wintergefieder, detaillierte Artenbeschreibungen erklären Merkmale, Vorkommen, Biologie, beschreiben den Ruf und das typische Verhalten all der Vogelarten, die sich im Winter gern in Gärten und Parks aufhalten. Praktisch sind die Angaben zu Nahrung und dem geeigneten Futter für die jeweilige Vogelart. Das Buch zeigt, was und wann gefüttert werden darf und gibt Tipps, wie man den gefiederten Freunden helfen kann, die kalte Jahreszeit gut zu überstehen. Was außerdem überzeugt: das handliche Format, der wetterfeste Einband und der günstige Preis. 